\title{
Common fixed point results for $\alpha-\psi$-contractions on a metric space endowed with graph
}

\author{
Nawab Hussain ${ }^{1 *}$, Muhammad Arshad ${ }^{2}$, Abdullah Shoaib² and Fahimuddin²
}

"Correspondence:

nhusain@kau.edu.sa

${ }^{1}$ Department of Mathematics, King

Abdulaziz University, P.O. Box 80203

Jeddah, 21589, Saudi Arabia

Full list of author information is

available at the end of the article

\section{空 Springer}

\begin{abstract}
Abdeljawad (Fixed Point Theory Appl., 2013:19) introduced the concept of $\alpha$-admissible for a pair of mappings. More recently Salimi et al. [Fixed Point Theory Appl., 2013:151] modified the notion of $\alpha$ - $\psi$-contractive mappings. In this paper we introduce the concept of an $\alpha$-admissible map with respect to $\eta$ and modify the $\alpha$ - $\psi$-contractive condition for a pair of mappings and establish common fixed point results for two, three, and four mappings in a closed ball in complete dislocated metric spaces. As an application, we derive some new common fixed point theorems for $\psi$-graphic contractions defined on dislocated metric space endowed with a graph as well as preordered dislocated metric space. Some comparative examples are constructed which illustrate the superiority of our results to the existing ones in the literature.
\end{abstract}

MSC: $46 \mathrm{~S} 40 ; 47 \mathrm{H} 10 ; 54 \mathrm{H} 25$

Keywords: common fixed point; complete dislocated metric space; $\alpha-\psi$-contractive mappings; $\psi$-graphic contractions; closed ball

\section{Introduction and preliminaries}

Fixed point results of mappings satisfying certain contractive condition on the entire domain has been at the center of rigorous research activities, for example, see [1-33]. From application point of view the situation is not yet completely satisfactory because it frequently happens that a mapping $T$ is a contraction not on the entire space $X$ but merely on a subset $Y$ of $X$. Recently Arshad et al. [8] proved a result concerning the existence of fixed points of a mapping satisfying a contractive condition on closed ball in a complete dislocated metric space (see also $[9,14,15,25,33])$. The notion of dislocated topologies has useful applications in the context of logic programming semantics (see [5, 17, 29]).

The existence of fixed points of $\alpha-\psi$-contractive and $\alpha$-admissible mappings in complete metric spaces has been studied by several researchers (see [18-20] and references therein). In this paper we discuss common fixed point results for $\alpha$ - $\psi$-contractive type mappings in a closed ball in complete dislocated metric space. Our results improve several well known recent conventional results in $[2,8,31]$. We also derive some new common fixed point theorems for $\psi$-graphic contractions as well as ordered contractions on preordered metric space. We give examples which show how these results can be used when the corresponding results cannot. 
Consistent with $[2,7,8,17,31]$, the following definitions and results will be needed in the sequel.

Definition 1.1 [17] Let $X$ be a non-empty set and let $d_{l}: X \times X \rightarrow[0, \infty)$ be a function, called a dislocated metric (or simply $d_{l}$-metric) if the following conditions hold for any $x, y, z \in X:$

(i) if $d_{l}(x, y)=0$, then $x=y$;

(ii) $d_{l}(x, y)=d_{l}(y, x)$;

(iii) $d_{l}(x, y) \leq d_{l}(x, z)+d_{l}(z, y)$.

The pair $\left(X, d_{l}\right)$ is then called a dislocated metric space. It is clear that if $d_{l}(x, y)=0$, then from (i), $x=y$. But if $x=y, d_{l}(x, y)$ may not be 0 .

Definition 1.2 [17] A sequence $\left\{x_{n}\right\}$ in a $d_{l}$-metric space $\left(X, d_{l}\right)$ is called a Cauchy sequence if given $\varepsilon>0$, there corresponds $n_{0} \in N$ such that for all $n, m \geq n_{0}$ we have $d_{l}\left(x_{m}, x_{n}\right)<\varepsilon$.

Definition 1.3 [17] A sequence $\left\{x_{n}\right\}$ in $d_{l}$-metric space converges with respect to $d_{l}$ if there exists $x \in X$ such that $d_{l}\left(x_{n}, x\right) \rightarrow 0$ as $n \rightarrow \infty$. In this case, $x$ is called a limit of $\left\{x_{n}\right\}$ and we write $x_{n} \rightarrow x$.

Definition 1.4 [17] A $d_{l}$-metric space $\left(X, d_{l}\right)$ is called complete if every Cauchy sequence in $X$ converges to a point in $X$.

Definition 1.5 Let $X$ be a non-empty set and $T, f: X \rightarrow X$. A point $y \in X$ is called point of coincidence of $T$ and $f$ if there exists a point $x \in X$ such that $y=T x=f x$, here $x$ is called coincidence point of $T$ and $f$. The mappings $T, f$ are said to be weakly compatible if they commute at their coincidence point (i.e., $T f x=f T x$ whenever $T x=f x$ ).

We require the following lemmas for subsequent use.

Lemma 1.6 [8] Let $X$ be a non-empty set and $f: X \rightarrow X$ be a function. Then there exists $E \subset X$ such that $f E=f X$ and $f: E \rightarrow X$ is one-to-one.

Lemma 1.7 [7] Let $X$ be a non-empty set and the mappings $S, T, f: X \rightarrow X$ have a unique point of coincidence $v$ in $X$. If $(S, f)$ and $(T, f)$ are weakly compatible, then $S, T, f$ have a unique common fixed point.

Let $\Psi$ denote the family of all nondecreasing functions $\psi:[0,+\infty) \rightarrow[0,+\infty)$ such that $\sum_{n=1}^{+\infty} \psi^{n}(t)<+\infty$ for all $t>0$, where $\psi^{n}$ is the $n$th iterate of $\psi$.

Lemma 1.8 [31] If $\psi \in \Psi$, then $\psi(t)<t$ for all $t>0$.

Definition 1.9 [2] Let $S, T: X \rightarrow X$ and $\alpha: X \times X \rightarrow[0,+\infty)$. We say that the pair $(S, T)$ is $\alpha$-admissible if $x, y \in X$ such that $\alpha(x, y) \geq 1$, then we have $\alpha(S x, T y) \geq 1$ and $\alpha(T x, S y) \geq 1$.

Definition 1.10 [31] Let $T: X \rightarrow X$ and $\alpha, \eta: X \times X \rightarrow[0,+\infty)$ two functions. We say that $T$ is $\alpha$-admissible mapping with respect to $\eta$ if $x, y \in X$ such that $\alpha(x, y) \geq \eta(x, y)$, then we 
have $\alpha(T x, T y) \geq \eta(T x, T y)$. Note that if we take $\eta(x, y)=1$, then $T$ is called an $\alpha$-admissible mapping [32].

\section{Common fixed point results in dislocated metric space}

We first extend the concept of $\alpha-\eta$-admissibility for the pair of mappings.

Definition 2.1 Let $S, T: X \rightarrow X$ and $\alpha, \eta: X \times X \rightarrow[0,+\infty)$ two functions. We say that the pair $(S, T)$ is $\alpha$-admissible with respect to $\eta$ if $x, y \in X$ such that $\alpha(x, y) \geq \eta(x, y)$, then we have $\alpha(S x, T y) \geq \eta(S x, T y)$ and $\alpha(T x, S y) \geq \eta(T x, S y)$. Also, if we take $\eta(x, y)=1$, then the pair $(S, T)$ is called $\alpha$-admissible, if we take, $\alpha(x, y)=1$, then we say that the pair $(S, T)$ is $\eta$-subadmissible mapping. If we take $\eta(x, y)=1$, then we obtain Definition 1 of Abdeljawad [2]. Also, if we take $S=T$, we obtain Definition 1.10.

Theorem 2.2 Let $\left(X, d_{l}\right)$ be a complete dislocated metric space and $S, T: X \rightarrow X$ be two mappings. Suppose there exist two functions, $\alpha, \eta: X \times X \rightarrow[0,+\infty)$ such that the pair $(S, T)$ is $\alpha$-admissible with respect to $\eta$. For $r>0, x_{0} \in \overline{B\left(x_{0}, r\right)}$, and $\psi \in \Psi$, assume that

$$
x, y \in \overline{B\left(x_{0}, r\right)}, \quad \alpha(x, y) \geq \eta(x, y) \quad \Longrightarrow \quad d_{l}(S x, T y) \leq \psi\left(d_{l}(x, y)\right)
$$

and

$$
\sum_{i=0}^{j} \psi^{i}\left(d_{l}\left(x_{0}, S x_{0}\right)\right) \leq r, \quad \text { for all } j \in \mathbb{N} .
$$

Suppose that the following assertions hold:

(i) $\alpha\left(x_{0}, S x_{0}\right) \geq \eta\left(x_{0}, S x_{0}\right)$;

(ii) for any sequence $\left\{x_{n}\right\}$ in $\overline{B\left(x_{0}, r\right)}$ such that $\alpha\left(x_{n}, x_{n+1}\right) \geq \eta\left(x_{n}, x_{n+1}\right)$ for all $n \in N \cup\{0\}$ and $x_{n} \rightarrow u \in \overline{B\left(x_{0}, r\right)}$ as $n \rightarrow+\infty$ then $\alpha\left(x_{n}, u\right) \geq \eta\left(x_{n}, u\right)$ for all $n \in \mathbb{N} \cup\{0\}$.

Then there exists a point $x^{*}$ in $\overline{B\left(x_{0}, r\right)}$ such that $x^{*}=S x^{*}=T x^{*}$.

Proof Let $x_{1}$ in $X$ be such that $x_{1}=S x_{0}$ and $x_{2}=T x_{1}$. Continuing this process, we construct a sequence $x_{n}$ of points in $X$ such that

$$
x_{2 i+1}=S x_{2 i}, \quad \text { and } \quad x_{2 i+2}=T x_{2 i+1}, \quad \text { where } i=0,1,2, \ldots
$$

By assumption $\alpha\left(x_{0}, x_{1}\right) \geq \eta\left(x_{0}, x_{1}\right)$ and the pair $(S, T)$ is $\alpha$-admissible with respect to $\eta$, we have, $\alpha\left(S x_{0}, T x_{1}\right) \geq \eta\left(S x_{0}, T x_{1}\right)$ from which we deduce that $\alpha\left(x_{1}, x_{2}\right) \geq \eta\left(x_{1}, x_{2}\right)$ which also implies that $\alpha\left(T x_{1}, S x_{2}\right) \geq \eta\left(T x_{1}, S x_{2}\right)$. Continuing in this way we obtain $\alpha\left(x_{n}, x_{n+1}\right) \geq$ $\eta\left(x_{n}, x_{n+1}\right)$ for all $n \in \mathbb{N} \cup\{0\}$. First, we show that $x_{n} \in \overline{B\left(x_{0}, r\right)}$ for all $n \in \mathbb{N}$. Using inequality (2), we have

$$
d_{l}\left(x_{0}, S x_{0}\right) \leq r
$$

It follows that

$$
x_{1} \in \overline{B\left(x_{0}, r\right)} \text {. }
$$


Let $x_{2}, \ldots, x_{j} \in \overline{B\left(x_{0}, r\right)}$ for some $j \in \mathbb{N}$. If $j=2 i+1$, where $i=0,1,2, \ldots \frac{j-1}{2}$ then using inequality (1), we obtain

$$
\begin{aligned}
d_{l}\left(x_{2 i+1}, x_{2 i+2}\right) & =d_{l}\left(S x_{2 i}, T x_{2 i+1}\right) \\
& \leq \psi\left(d_{l}\left(x_{2 i}, x_{2 i+1}\right)\right) \\
& \leq \psi^{2}\left(d_{l}\left(x_{2 i-1}, x_{2 i}\right)\right) \\
& \leq \cdots \leq \psi^{2 i+1}\left(d_{l}\left(x_{0}, x_{1}\right)\right) .
\end{aligned}
$$

Thus we have

$$
d_{l}\left(x_{2 i+1}, x_{2 i+2}\right) \leq \psi^{2 i+1}\left(d_{l}\left(x_{0}, x_{1}\right)\right)
$$

If $j=2 i+2$, then as $x_{1}, x_{2}, \ldots, x_{j} \in \overline{B\left(x_{0}, r\right)}$ where $\left(i=0,1,2, \ldots, \frac{j-2}{2}\right)$, we obtain,

$$
d_{l}\left(x_{2 i+2}, x_{2 i+3}\right) \leq \psi^{2(i+1)}\left(d_{l}\left(x_{0}, x_{1}\right)\right)
$$

Thus from inequality (3) and (4), we have

$$
d_{l}\left(x_{j}, x_{j+1}\right) \leq \psi^{j}\left(d_{l}\left(x_{0}, x_{1}\right)\right)
$$

Now,

$$
\begin{aligned}
d_{l}\left(x_{0}, x_{j+1}\right) & =d_{l}\left(x_{0}, x_{1}\right)+d_{l}\left(x_{1}, x_{2}\right)+d_{l}\left(x_{2}, x_{3}\right)+\cdots+d_{l}\left(x_{j}, x_{j+1}\right) \\
& \leq \sum_{i=0}^{j} \psi^{i}\left(d_{l}\left(x_{0}, x_{1}\right)\right) \\
& \leq r .
\end{aligned}
$$

Thus $x_{j+1} \in \overline{B\left(x_{0}, r\right)}$. Hence $x_{n} \in \overline{B\left(x_{0}, r\right)}$ for all $n \in \mathbb{N}$. Now inequality (5) can be written as

$$
d_{l}\left(x_{n}, x_{n+1}\right) \leq \psi^{n}\left(d_{l}\left(x_{0}, x_{1}\right)\right), \quad \text { for all } n \in \mathbb{N} \text {. }
$$

Fix $\varepsilon>0$ and let $n(\varepsilon) \in \mathbb{N}$ such that $\sum \psi^{n}\left(d_{l}\left(x_{0}, x_{1}\right)\right)<\varepsilon$. Let $n, m \in \mathbb{N}$ with $m>n>k(\varepsilon)$, then by using the triangle inequality, we obtain

$$
\begin{aligned}
d_{l}\left(x_{n}, x_{m}\right) & \leq \sum_{k=n}^{m-1} d_{l}\left(x_{k}, x_{k+1}\right) \leq \sum_{k=n}^{m-1} \psi^{k}\left(d_{l}\left(x_{0}, x_{1}\right)\right) \\
& \leq \sum_{n \geq n(\varepsilon)} \psi^{k}\left(d_{l}\left(x_{0}, x_{1}\right)\right)<\varepsilon
\end{aligned}
$$

Thus we proved that $\left\{x_{n}\right\}$ is a Cauchy sequence in $\left(\overline{B\left(x_{0}, r\right)}, d_{l}\right)$. As every closed ball in a complete dislocated metric space is complete, so there exists $x^{*} \in \overline{B\left(x_{0}, r\right)}$ such that $x_{n} \rightarrow$ $x^{*}$. Also

$$
\lim _{n \rightarrow \infty} d_{l}\left(x_{n}, x^{*}\right)=0
$$


On the other hand, from (ii), we have

$$
\alpha\left(x^{*}, x_{n}\right) \geq \eta\left(x^{*}, x_{n}\right) \quad \text { for all } n \in \mathbb{N} \cup\{0\}
$$

Now using the triangle inequality, together with (1) and (8), we get

$$
d_{l}\left(S x^{*}, x_{2 i+2}\right) \leq \psi\left(d_{l}\left(x^{*}, x_{2 i+1}\right)\right)<d_{l}\left(x^{*}, x_{2 i+1}\right) .
$$

Letting $i \rightarrow \infty$ and by using inequality (7), we obtain $d_{l}\left(S x^{*}, x^{*}\right)<0$. Hence $S x^{*}=x^{*}$. Similarly by using

$$
d_{l}\left(T x^{*}, x_{2 i+1}\right) \leq \psi\left(d_{l}\left(x^{*}, x_{2 i}\right)\right)<d_{l}\left(x^{*}, x_{2 i}\right),
$$

we obtain $d_{l}\left(T x^{*}, x^{*}\right)=0$, that is, $T x^{*}=x^{*}$. Hence $S$ and $T$ have a common fixed point in $\overline{B\left(x_{0}, r\right)}$.

If $\eta(x, y)=1$ for all $x, y \in X$ in Theorem 2.2, we obtain the following result.

Corollary 2.3 Let $\left(X, d_{l}\right)$ be a complete dislocated metric space and $S, T: X \rightarrow X, r>0$ and $x_{0}$ be an arbitrary point in $\overline{B\left(x_{0}, r\right)}$. Suppose there exists $\alpha: X \times X \rightarrow[0,+\infty)$ such that the pair $(S, T)$ is $\alpha$-admissible. For $\psi \in \Psi$, assume that

$$
x, y \in \overline{B\left(x_{0}, r\right)}, \quad \alpha(x, y) \geq 1 \quad \Longrightarrow \quad d_{l}(S x, T y) \leq \psi\left(d_{l}(x, y)\right)
$$

and

$$
\sum_{i=0}^{j} \psi^{i}\left(d_{l}\left(x_{0}, S x_{0}\right)\right) \leq r \quad \text { for all } j \in \mathbb{N} \text {. }
$$

Suppose that the following assertions hold:

(i) $\alpha\left(x_{0}, S x_{0}\right) \geq 1$;

(ii) for any sequence $\left\{x_{n}\right\}$ in $\overline{B\left(x_{0}, r\right)}$ such that $\alpha\left(x_{n}, x_{n+1}\right) \geq 1$ for all $n \in \mathbb{N} \cup\{0\}$ and $x_{n} \rightarrow u \in \overline{B\left(x_{0}, r\right)}$ as $n \rightarrow+\infty$ then $\alpha\left(x_{n}, u\right) \geq 1$ for all $n \in \mathbb{N} \cup\{0\}$.

Then there exists a point $x^{*}$ in $\overline{B\left(x_{0}, r\right)}$ such that $x^{*}=S x^{*}=T x^{*}$.

If $\alpha(x, y)=1$ for all $x, y \in X$ in Theorem 2.2, we obtain following result.

Corollary 2.4 Let $\left(X, d_{l}\right)$ be a complete dislocated metric space and $S, T: X \rightarrow X$ be two mappings. Suppose there exists $\eta: X \times X \rightarrow[0,+\infty)$ such that the pair $(S, T)$ is $\eta$ subadmissible. For $\psi \in \Psi$ and $x_{0} \in \overline{B\left(x_{0}, r\right)}$, assume that

$$
x, y \in \overline{B\left(x_{0}, r\right)}, \quad \eta(x, y) \leq 1 \quad \Longrightarrow \quad d_{l}(S x, T y) \leq \psi\left(d_{l}(x, y)\right)
$$

and

$$
\sum_{i=0}^{j} \psi^{i}\left(d_{l}\left(x_{0}, S x_{0}\right)\right) \leq r \quad \text { for all } j \in \mathbb{N} \text {. }
$$

Suppose that the following assertions hold: 
(i) $\eta\left(x_{0}, S x_{0}\right) \leq 1$;

(ii) for any sequence $\left\{x_{n}\right\}$ in $\overline{B\left(x_{0}, r\right)}$ such that $\eta\left(x_{n}, x_{n+1}\right) \leq 1$ for all $n \in \mathbb{N} \cup\{0\}$ and $x_{n} \rightarrow u \in \overline{B\left(x_{0}, r\right)}$ as $n \rightarrow+\infty$ then $\eta\left(x_{n}, u\right) \leq 1$ for all $n \in \mathbb{N} \cup\{0\}$.

Then there exists a point $x^{*}$ in $\overline{B\left(x_{0}, r\right)}$ such that $x^{*}=S x^{*}=T x^{*}$.

Corollary 2.5 (Theorem 2.2 of [32]) Let $(X, d)$ be a complete metric space and $S: X \rightarrow X$ be an $\alpha$-admissible mapping. Assume that for $\psi \in \Psi$,

$$
\alpha(x, y) d(S x, S y) \leq \psi(d(x, y))
$$

holds for all $x, y \in X$. Also, suppose that the following assertions hold:

(i) there exists $x_{0} \in X$ such that $\alpha\left(x_{0}, S x_{0}\right) \geq 1$;

(ii) for any sequence $\left\{x_{n}\right\}$ in $X$ with $\alpha\left(x_{n}, x_{n+1}\right) \geq 1$ for all $n \in \mathbb{N} \cup\{0\}$ and $x_{n} \rightarrow x$ as $n \rightarrow+\infty$, we have $\alpha\left(x_{n}, x\right) \geq 1$ for all $n \in \mathbb{N} \cup\{0\}$.

Then $S$ has a fixed point.

Theorem 2.6 On adding the condition 'if $x^{*}$ is any common fixed point in $\overline{B\left(x_{0}, r\right)}$ of S and $T, x$ be any fixed point of $S$ or $T$ in $\overline{B\left(x_{0}, r\right)}$, then $\alpha\left(x, x^{*}\right) \geq \eta\left(x, x^{*}\right)^{\prime}$ to the hypotheses of Theorem 2.2, S and T have a unique common fixed point $x^{*}$ and $d_{l}\left(x^{*}, x^{*}\right)=0$.

Proof Assume that $y^{*}$ be another fixed point of $T$ in $\overline{B\left(x_{0}, r\right)}$, then, by assumption, $\alpha\left(x^{*}, y^{*}\right) \geq \eta\left(x^{*}, y^{*}\right)$,

$$
d_{l}\left(x^{*}, y^{*}\right)=d_{l}\left(S x^{*}, T y^{*}\right) \leq \psi\left(d_{l}\left(x^{*}, y^{*}\right)\right) .
$$

A contradiction to the fact that for each $t>0, \psi(t)<t$. So $x^{*}=y^{*}$. Hence $T$ has no fixed point other than $x^{*}$. Similarly, $S$ has no fixed point other than $x^{*}$. Now, $\alpha\left(x^{*}, x^{*}\right) \geq \eta\left(x^{*}, x^{*}\right)$, then

$$
d_{l}\left(x^{*}, x^{*}\right)=d_{l}\left(S x^{*}, T x^{*}\right) \leq \psi\left(d_{l}\left(x^{*}, x^{*}\right)\right) .
$$

This implies that

$$
d_{l}\left(x^{*}, x^{*}\right)=0
$$

Example 2.7 Let $X=Q^{+} \cup\{0\}$ and $d_{l}: X \times X \rightarrow X$ be defined by $d_{l}(x, y)=x+y$. Then $\left(X, d_{l}\right)$ is complete dislocated metric space (see [8]). Let $S, T: X \rightarrow X$ be defined by

$$
S x= \begin{cases}\frac{x}{7} & \text { if } x \in[0,1] \cap X, \\ x-\frac{1}{2} & \text { if } x \in(1, \infty) \cap X,\end{cases}
$$

and

$$
T x= \begin{cases}\frac{2 x}{7} & \text { if } x \in[0,1] \cap X, \\ x-\frac{1}{3} & \text { if } x \in(1, \infty) \cap X .\end{cases}
$$


Considering, $x_{0}=1, r=2, \psi(t)=\frac{t}{3}$ and $\alpha(x, y)=2$. Now $\overline{B\left(x_{0}, r\right)}=[0,1] \cap X$. Also,

$$
\begin{aligned}
& d_{l}\left(x_{0}, S x_{0}\right)=d_{l}(1, S 1)=d_{l}\left(1, \frac{1}{7}\right)=1+\frac{1}{7}=\frac{8}{7}, \\
& \sum_{i=0}^{n} \psi^{n}\left(d_{l}\left(x_{0}, S x_{0}\right)\right)=\frac{8}{7} \sum_{i=0}^{n} \frac{1}{3^{n}}<\frac{3}{2}\left(\frac{8}{7}\right)=\frac{12}{7}<2 .
\end{aligned}
$$

Also if $x, y \in(1, \infty) \cap X$, then

$$
\begin{aligned}
& 3 x+3 y-\frac{5}{2}>x+y, \\
& x+y-\frac{5}{6}>\frac{x+y}{3}, \\
& x+y-\frac{5}{6}>\psi(x+y), \\
& d_{l}(S x, T y)>\psi\left(d_{l}(x, y)\right) .
\end{aligned}
$$

Then the contractive condition does not hold on $X$. Also, if $x, y \in \overline{B\left(x_{0}, r\right)}$, then

$$
\begin{aligned}
& \frac{3 x}{7}+\frac{6 y}{7} \leq x+y, \\
& \frac{x}{7}+\frac{2 y}{7} \leq \frac{x+y}{3}, \\
& \frac{x}{7}+\frac{2 y}{7} \leq \psi(x+y), \\
& d_{l}(S x, T y) \leq \psi\left(d_{l}(x, y)\right) .
\end{aligned}
$$

Therefore, all the conditions of Corollary 2.3 are satisfied and $S$ and $T$ have a common fixed point 0 .

Now we apply our Theorem 2.6 to obtain unique common fixed point of three mappings on a closed ball in complete dislocated metric space.

Theorem 2.8 Let $\left(X, d_{l}\right)$ be a dislocated metric space, $S, T, f: X \rightarrow X$ such that $S X \cup T X \subset$ $f X, r>0$ and $x_{0} \in \overline{B\left(x_{0}, r\right)}$. Suppose there exist two functions, $\alpha, \eta: X \times X \rightarrow[0,+\infty) \alpha-$ admissible with respect to $\eta$ and $\psi \in \Psi$ such that

$$
\text { for all } f x, f y \in \overline{B\left(f x_{0}, r\right)}, \quad \alpha(f x, f y) \geq \eta(f x, f y) \quad \Longrightarrow \quad d_{l}(S x, T y) \leq \psi\left(d_{l}(f x, f y)\right)
$$

and

$$
\sum_{i=0}^{j} \psi^{i}\left(d_{l}\left(f x_{0}, S x_{0}\right)\right) \leq r \quad \text { for all } j \in \mathbb{N}
$$

\section{Suppose that}

(i) the pair $(S, T)$ and $f$ are $\alpha$-admissible with respect to $\eta$;

(ii) $\alpha\left(f x_{0}, S x_{0}\right) \geq \eta\left(f x_{0}, S x_{0}\right)$; 
(iii) if $\left\{x_{n}\right\}$ is a sequence in $\overline{B\left(f x_{0}, r\right)}$ such that $\alpha\left(x_{n}, x_{n+1}\right) \geq \eta\left(x_{n}, x_{n+1}\right)$ for all $n$ and $x_{n} \rightarrow u \in \overline{B\left(f x_{0}, r\right)}$ as $n \rightarrow+\infty$ then $\alpha\left(x_{n}, u\right) \geq \eta\left(x_{n}, u\right)$ for all $n \in \mathbb{N} \cup\{0\}$;

(iv) if $f x$ is any point in $\overline{B\left(f x_{0}, r\right)}$ such that $S x=T x=f x$ and fy be any point in $\overline{B\left(f x_{0}, r\right)}$ such that $S y=f y$ or $T y=f y$, then $\alpha(f x, f y) \geq \eta(f x, f y)$;

(v) $f X$ is complete subspace of $X$ and $(S, f)$ and $(T, f)$ are weakly compatible.

Then $S, T$, and $f$ have a unique common fixed point $f z$ in $\overline{B\left(f x_{0}, r\right)}$. Moreover, $d_{l}(f z, f z)=0$.

Proof By Lemma 1.6, there exists $E \subset X$ such that $f E=f X$ and $f: E \rightarrow X$ is one-to-one. Now since $S X \cup T X \subset f X$, we define two mappings $g, h: f E \rightarrow f E$ by $g(f x)=S x$ and $h(f x)=$ $T x$, respectively. Since $f$ is one-to-one on $E$, then $g, h$ are well defined. Now $f x_{0} \in \overline{B\left(f x_{0}, r\right)} \subseteq$ $f X$. Then $f x_{0} \in f X$. Let $y_{0}=f x_{0}$, choose a point $y_{1}$ in $f X$ such that $y_{1}=g\left(y_{0}\right)$ and let $y_{2}=h\left(y_{1}\right)$. Continuing this process and having chosen $y_{n}$ in $f X$ such that

$$
y_{2 i+1}=g\left(y_{2 i}\right) \quad \text { and } \quad y_{2 i+2}=h\left(y_{2 i+1}\right), \quad \text { where } i=0,1,2, \ldots
$$

As $f$ is $\alpha$-admissible then $\alpha(x, y) \geq \eta(x, y)$ implies

$$
\alpha(f x, f y) \geq \eta(f x, f y) .
$$

Also if $(S, T)$ is $\alpha$-admissible then $\alpha(x, y) \geq \eta(x, y)$ implies

$$
\begin{aligned}
& \alpha(S x, T y)=\alpha(g(f x), h(f y)) \geq \eta(g(f x), h(f y)) \quad \text { and } \\
& \alpha(h(f x), g(f y)) \geq \eta(h(f x), g(f y)) .
\end{aligned}
$$

This implies that the pair $(g, h)$ is $\alpha$-admissible. As $\alpha\left(y_{0}, y_{1}\right) \geq \eta\left(y_{0}, y_{1}\right) \Longrightarrow \alpha\left(g y_{0}, h y_{1}\right) \geq$ $\eta\left(g y_{0}, h y_{1}\right) \Longrightarrow \alpha\left(h y_{1}, g y_{2}\right) \geq \eta\left(h y_{1}, g y_{2}\right)$. Continuing this process, we have $\alpha\left(y_{n}, y_{n+1}\right) \geq$ $\eta\left(y_{n}, y_{n+1}\right)$. Following similar arguments to those of Theorem 2.2, $y_{n} \in \overline{B\left(f x_{0}, r\right)}$. Also by inequality (10).

$$
\sum_{i=0}^{j} \psi^{i}\left(d_{l}\left(y_{0}, g y_{0}\right)\right) \leq r \quad \text { for all } j \in N
$$

Note that for $f x, f y \in \overline{B\left(f x_{0}, r\right)}$ and $\alpha(f x, f y) \leq \eta(f x, f y)$. Then by using inequality (9), we have

$$
d_{l}(g(f x), h(f y)) \leq \psi\left(d_{l}(f x, f y)\right) .
$$

As $f X$ is a complete space, all conditions of Theorem 2.6 are satisfied, we deduce that there exists a unique common fixed point $f z \in \overline{B\left(f x_{0}, r\right)}$ of $g$ and $h$. Now $f z=g(f z)=h(f z)$ or $f z=S z=T z=f z$. Thus $f z$ is the point of coincidence of $S, T$ and $f$. Let $v \in \overline{B\left(f x_{0}, r\right)}$ be another point of coincidence of $f, S$ and $T$ then there exists $u \in \overline{B\left(f x_{0}, r\right)}$ such that $v=f u=S u=T u$, which implies that $f u=g(f u)=h(f u)$. A contradiction as $f z \in \overline{B\left(f x_{0}, r\right)}$ is a unique common fixed point of $g$ and $h$. Hence $v=f z$. Thus $S, T$ and $f$ have a unique point of coincidence $f z \in \overline{B\left(f x_{0}, r\right)}$. Now since $(S, f)$ and $(T, f)$ are weakly compatible, by Lemma $1.7 \mathrm{fz}$ is a unique common fixed point of $S, T$, and $f$. 
Similarly, we can apply our Theorem 2.6 to obtain unique common fixed point and point of coincidence of four mappings in complete dislocated metric space. One can easily obtain conclusion by using the technique given in the proof of Theorem 2.8 [8].

Theorem 2.9 Let $\left(X, d_{l}\right)$ be a dislocated metric space and $S, T, g$ and $f$ be self-mappings on $X$ such that $S X, T X \subset f X=g X, r>0$ and $x_{0} \in \overline{B\left(x_{0}, r\right)}$. Suppose there exist two functions $\alpha, \eta: X \times X \rightarrow[0,+\infty)$ is $\alpha$-admissible with respect to $\eta$ and $\psi \in \Psi$ such that

$$
\text { for all } f x, g y \in \overline{B\left(f x_{0}, r\right)}, \quad \alpha(f x, g y) \leq \eta(f x, g y) \quad \Longrightarrow \quad d_{l}(S x, T y) \leq \psi\left(d_{l}(f x, g y)\right)
$$

and

$$
\sum_{i=0}^{j} \psi^{i}\left(d_{l}\left(f x_{0}, S x_{0}\right)\right) \leq r \quad \text { for all } j \in \mathbb{N} .
$$

\section{Suppose that}

(i) the pairs $(S, T)$ and $(f, g)$ are $\alpha$-admissible with respect to $\eta$;

(ii) $\alpha\left(f x_{0}, S x_{0}\right) \geq \eta\left(f x_{0}, S x_{0}\right)$;

(iii) if $\left\{x_{n}\right\}$ is a sequence in $\overline{B\left(f x_{0}, r\right)}$ such that $\alpha\left(x_{n}, x_{n+1}\right) \geq \eta\left(x_{n}, x_{n+1}\right)$ for all $n$ and

$$
x_{n} \rightarrow u \in \overline{B\left(f x_{0}, r\right)} \text { as } n \rightarrow+\infty \text { then } \alpha\left(x_{n}, u\right) \geq \eta\left(x_{n}, u\right) \text { for all } n \text {; }
$$

(iv) if $f x=g x$ is any point in $\overline{B\left(f x_{0}, r\right)}$ such that $S x=T x=f x$ and $f y=g y$ be any point in $\overline{B\left(f x_{0}, r\right)}$ such that $S y=f y$ or $T y=f y$, then $\alpha(f x, f y) \geq \eta(f x, f y)$;

(v) $f X$ is complete subspace of $X$ and $(S, f)$ and $(T, g)$ are weakly compatible.

Then $S, T, f$, and $g$ have a unique common fixed point $f z$ in $\overline{B\left(f x_{0}, r\right)}$.

A partial metric version of Theorem 2.2 is given below.

Theorem 2.10 Let $(X, p)$ be a complete partial metric space, $S, T: X \rightarrow X$ be two maps, $r>0$ and $x_{0} \in \overline{B\left(x_{0}, r\right)}$. Suppose there exist two functions, $\alpha, \eta: X \times X \rightarrow[0,+\infty)$ such that $(S, T)$ be $\alpha$-admissible with respect to $\eta$ and $\psi \in \Psi$. Assume that

$$
x, y \in \overline{B\left(x_{0}, r\right)}, \quad \alpha(x, y) \geq \eta(x, y) \quad \Longrightarrow \quad p(S x, T y) \leq \psi(p(x, y))
$$

and

$$
\sum_{i=0}^{j} \psi^{i}\left(p\left(x_{0}, S x_{0}\right)\right) \leq r+p\left(x_{0}, x_{0}\right) \quad \text { for all } j \in \mathbb{N} .
$$

Suppose that the following assertions hold:

(i) $\alpha\left(x_{0}, S x_{0}\right) \geq \eta\left(x_{0}, S x_{0}\right)$;

(ii) for any sequence $\left\{x_{n}\right\}$ in $\overline{B\left(x_{0}, r\right)}$ such that $\alpha\left(x_{n}, x_{n+1}\right) \geq \eta\left(x_{n}, x_{n+1}\right)$ for all $n \in N \cup\{0\}$ and $x_{n} \rightarrow u \in \overline{B\left(x_{0}, r\right)}$ as $n \rightarrow+\infty$ then $\alpha\left(x_{n}, u\right) \geq \eta\left(x_{n}, u\right)$ for all $n \in \mathbb{N} \cup\{0\}$.

Then there exists a point $x^{*}$ in $\overline{B\left(x_{0}, r\right)}$ such that $x^{*}=S x^{*}=T x^{*}$.

\section{Fixed point results for graphic contractions in dislocated metric spaces}

Consistent with Jachymski [24], let $\left(X, d_{l}\right)$ be a dislocated metric space and $\Delta$ denotes the diagonal of the Cartesian product $X \times X$. Consider a directed graph $G$ such that the 
set $V(G)$ of its vertices coincides with $X$, and the set $E(G)$ of its edges contains all loops, i.e., $E(G) \supseteq \Delta$. We assume $G$ has no parallel edges, so we can identify $G$ with the pair $(V(G), E(G))$. Moreover, we may treat $G$ as a weighted graph (see [24]) by assigning to each edge the distance between its vertices. If $x$ and $y$ are vertices in a graph $G$, then a path in $G$ from $x$ to $y$ of length $m(m \in \mathbb{N})$ is a sequence $\left\{x_{i}\right\}_{i=0}^{m}$ of $m+1$ vertices such that $x_{0}=x, x_{m}=y$ and $\left(x_{n-1}, x_{n}\right) \in E(G)$ for $i=1, \ldots, m$. A graph $G$ is connected if there is a path between any two vertices. $G$ is weakly connected if $\tilde{G}$ is connected (see for details $[1,11$, $21,24])$.

Definition 3.1 [24] We say that a mapping $T: X \rightarrow X$ is a Banach $G$-contraction or simply $G$-contraction if $T$ preserves the edges of $G$, i.e.,

$$
\forall x, y \in X, \quad(x, y) \in E(G) \quad \Rightarrow \quad(T x, T y) \in E(G)
$$

and $T$ decreases the weights of the edges of $G$ in the following way:

$$
\exists k \in(0,1), \forall x, y \in X, \quad(x, y) \in E(G) \quad \Rightarrow \quad d(T x, T y) \leq k d(x, y) .
$$

Now we extend the concept of $G$-contraction for the pair of maps as follows.

Definition 3.2 Let $\left(X, d_{l}\right)$ be a dislocated metric space endowed with a graph $G$ and $S, T: X \rightarrow X$ be self-mappings. Assume that for $r>0, x_{0} \in \overline{B\left(x_{0}, r\right)}$ and $\psi \in \Psi$ following conditions hold:

$$
\begin{array}{ll}
\forall x, y \in \overline{B\left(x_{0}, r\right)}, \quad(x, y) \in E(G) \quad \Rightarrow \quad(S x, T y) \in E(G) \quad \text { and } \quad(T x, S y) \in E(G) \\
\forall x, y \in \overline{B\left(x_{0}, r\right),} \quad(x, y) \in E(G) \quad \Rightarrow \quad d_{l}(S x, T y) \leq \psi\left(d_{l}(x, y)\right) .
\end{array}
$$

Then the mappings $(S, T)$ are called a $\psi$-graphic contractive mappings. If $\psi(t)=k t$ for some $k \in[0,1)$, then we say $(S, T)$ are $G$-contractive mappings.

Theorem 3.3 Let $\left(X, d_{l}\right)$ be a complete dislocated metric space endowed with a graph $G$ and $S, T: X \rightarrow X$ be $\psi$-graphic contractive mappings and $x_{0} \in \overline{B\left(x_{0}, r\right)}$. Suppose that the following assertions hold:

(i) $\left(x_{0}, S x_{0}\right) \in E(G)$ and $\sum_{i=0}^{j} \psi^{i}\left(d_{l}\left(x_{0}, S x_{0}\right)\right) \leq r$ for all $j \in \mathbb{N}$;

(ii) if $\left\{x_{n}\right\}$ is a sequence in $\overline{B\left(x_{0}, r\right)}$ such that $\left(x_{n}, x_{n+1}\right) \in E(G)$ for all $n \in \mathbb{N}$ and $x_{n} \rightarrow x$ as $n \rightarrow+\infty$, then $\left(x_{n}, x\right) \in E(G)$ for all $n \in \mathbb{N}$.

Then $S$ and $T$ have a common fixed point.

Proof Define, $\alpha: X^{2} \rightarrow(-\infty,+\infty)$ by $\alpha(x, y)=\left\{\begin{array}{ll}1, & \text { if }(x, y) \in E(G), \\ 0, & \text { otherwise. }\end{array}\right.$ At first we prove that the mappings $(S, T)$ are $\alpha$-admissible. Let $x, y \in \overline{B\left(x_{0}, r\right)}$ with $\alpha(x, y) \geq 1$, then $(x, y) \in E(G)$. As $(S, T)$ are $\psi$-graphic contractive mappings, we have $(S x, T y) \in E(G)$ and $(T x, S y) \in E(G)$. That is, $\alpha(S x, T y) \geq 1$ and $\alpha(T x, S y) \geq 1$. Thus $S, T$ are $\alpha$-admissible mappings. From (i) there exists $x_{0}$ such that $\left(x_{0}, S x_{0}\right) \in E(G)$. That is, $\alpha\left(x_{0}, S x_{0}\right) \geq 1$.

If $x, y \in \overline{B\left(x_{0}, r\right)}$ with $\alpha(x, y) \geq 1$, then $(x, y) \in E(G)$. Now, since $S, T$ are $\psi$-graphic contractive mappings, $d_{l}(S x, T y) \leq \psi\left(d_{l}(x, y)\right)$. That is,

$$
\alpha(x, y) \geq 1 \quad \Longrightarrow \quad d_{l}(S x, T y) \leq \psi\left(d_{l}(x, y)\right) .
$$


Let $\left\{x_{n}\right\} \subset \overline{B\left(x_{0}, r\right)}$ with $x_{n} \rightarrow x$ as $n \rightarrow \infty$ and $\alpha\left(x_{n}, x_{n+1}\right) \geq 1$ for all $n \in \mathbb{N}$. Then $\left(x_{n}, x_{n+1}\right) \in$ $E(G)$ for all $n \in \mathbb{N}$ and $x_{n} \rightarrow x$ as $n \rightarrow+\infty$. So by (ii) we have $\left(x_{n}, x\right) \in E(G)$ for all $n \in \mathbb{N}$. That is, $\alpha\left(x_{n}, x\right) \geq 1$. Hence, all conditions of Corollary 2.3 are satisfied and $S$ and $T$ have a common fixed point.

Theorem 3.2(2 $2^{\circ}$ [24] and Theorem 2.3(2) [12] are extended to $\psi$-graphic contractive pair defined on a dislocated metric space as follows.

Theorem 3.4 Let $\left(X, d_{l}\right)$ be a complete dislocated metric space endowed with a graph $G$ and $S, T: X \rightarrow X$ be $\psi$-graphic contractive mappings and $x_{0} \in \overline{B\left(x_{0}, r\right)}$. Suppose that the following assertions hold:

(i) $\left(x_{0}, S x_{0}\right) \in E(G)$ and $\sum_{i=0}^{j} \psi^{i}\left(d_{l}\left(x_{0}, S x_{0}\right)\right) \leq$ for all $j \in \mathbb{N}$;

(iis) $(x, z) \in E(G)$ and $(z, y) \in E(G)$ imply $(x, y) \in E(G)$ for all $x, y, z \in X$, that is, $E(G)$ is a quasi-order [24] and if $\left\{x_{n}\right\}$ is a sequence in $\overline{B\left(x_{0}, r\right)}$ such that $\left(x_{n}, x_{n+1}\right) \in E(G)$ for all $n \in \mathbb{N}$ and $x_{n} \rightarrow x$ as $n \rightarrow+\infty$, then there is a subsequence $\left\{x_{k_{n}}\right\}$ with $\left(x_{k_{n}}, x\right) \in E(G)$ for all $n \in \mathbb{N}$.

Then $S, T$ have a common fixed point.

Proof Condition (iis) implies that of (ii) in Theorem 3.3 (see Remark 3.1 [24]). Now the conclusion follows from Theorem 3.3.

Corollary 3.5 Let $\left(X, d_{l}\right)$ be a complete dislocated metric space endowed with a graph $G$ and $S, T: X \rightarrow X$ be two mappings and $x_{0} \in \overline{B\left(x_{0}, r\right)}$. Suppose that the following assertions hold:

(i) $(S, T)$ are $G$-contractive mappings;

(ii) $\left(x_{0}, S x_{0}\right) \in E(G)$ and $d_{l}\left(x_{0}, S x_{0}\right) \leq(1-k) r$;

(iii) if $\left\{x_{n}\right\}$ is a sequence in $\overline{B\left(x_{0}, r\right)}$ such that $\left(x_{n}, x_{n+1}\right) \in E(G)$ for all $n \in \mathbb{N}$ and $x_{n} \rightarrow x$ as $n \rightarrow+\infty$, then $\left(x_{n}, x\right) \in E(G)$ for all $n \in \mathbb{N}$.

Then $S$ and $T$ have a common fixed point.

Corollary 3.6 Let $\left(X, d_{l}\right)$ be a complete dislocated metric space endowed with a graph $G$ and $S: X \rightarrow X$ be a mapping and $x_{0} \in \overline{B\left(x_{0}, r\right)}$. Suppose that the following assertions hold:

(i) $S$ is Banach G-contraction on $\overline{B\left(x_{0}, r\right)}$;

(ii) $\left(x_{0}, S x_{0}\right) \in E(G)$ and $d_{l}\left(x_{0}, S x_{0}\right) \leq(1-k) r$;

(iii) if $\left\{x_{n}\right\}$ is a sequence in $\overline{B\left(x_{0}, r\right)}$ such that $\left(x_{n}, x_{n+1}\right) \in E(G)$ for all $n \in \mathbb{N}$ and $x_{n} \rightarrow x$ as $n \rightarrow+\infty$, then $\left(x_{n}, x\right) \in E(G)$ for all $n \in \mathbb{N}$.

Then $S$ has a fixed point.

Corollary 3.7 Let $\left(X, d_{l}\right)$ be a complete dislocated metric space endowed with a graph $G$ and $S: X \rightarrow X$ be a mapping. Suppose that the following assertions hold:

(i) $S$ is Banach G-contraction on $X$ and there is $x_{0} \in X$ such that $\left(x_{0}, S x_{0}\right) \in E(G)$;

(ii) if $\left\{x_{n}\right\}$ is a sequence in $X$ such that $\left(x_{n}, x_{n+1}\right) \in E(G)$ for all $n \in \mathbb{N}$ and $x_{n} \rightarrow x$ as $n \rightarrow+\infty$, then $\left(x_{n}, x\right) \in E(G)$ for all $n \in \mathbb{N}$.

Then $S$ has a fixed point.

The study of existence of fixed points in partially ordered sets has been initiated by Ran and Reurings [28] with applications to matrix equations. Agarwal, et al. [3, 4], Bhaskar and Lakshmikantham [10], Ciric et al. [13] and Hussain et al. [22, 23] presented some new 
results for nonlinear contractions in partially ordered metric spaces and noted that their theorems can be used to investigate a large class of problems. Roldán et al. [30] and Harandi et al. [6] proved some results in preordered metric spaces which is a generalization of partially ordered metric spaces. Here as an application of our results we deduce some new common fixed point results in preordered dislocated metric spaces.

Recall that if $(X, \preceq)$ is a preordered set and $T: X \rightarrow X$ is such that for $x, y \in X$, with $x \preceq y$ implies $T x \preceq T y$, then the mapping $T$ is said to be nondecreasing. If for $x, y \in X$, with $x \preceq y$ implies $S x \preceq T y$ and $T x \preceq S y$, then the pair $(S, T)$ is called jointly nondecreasing.

Let $X$ be a non-empty set. Then $\left(X, d_{l}, \preceq\right)$ is called a preordered dislocated metric space if $d_{l}$ is a dislocated metric on $X$ and $\preceq$ is a preorder on $X$. Let $\left(X, d_{l}, \preceq\right)$ be a preordered dislocated metric space. Define the graph $G$ by

$$
E(G):=\{(x, y) \in X \times X: x \preceq y\} .
$$

For this graph, the first condition in Definition 3.2 means $S, T$ are jointly nondecreasing with respect to this order. From Theorems 3.3-Corollary 3.7 we derive the following important results in preordered dislocated metric spaces.

Theorem 3.8 Let $\left(X, d_{l}, \preceq\right)$ be a preordered complete dislocated metric space and let the pair $(S, T)$ of self-maps of $X$ be jointly nondecreasing and $x_{0} \in \overline{B\left(x_{0}, r\right)}$. Suppose that the following assertions hold:

(i) for all $x, y \in \overline{B\left(x_{0}, r\right)}$, with $x \preceq y \Longrightarrow d_{l}(S x, T y) \leq \psi\left(d_{l}(x, y)\right)$;

(ii) $x_{0} \preceq S x_{0}$ and $\sum_{i=0}^{j} \psi^{i}\left(d_{l}\left(x_{0}, S x_{0}\right)\right) \leq r$ for all $j \in \mathbb{N}$;

(iii) if $\left\{x_{n}\right\}$ is a nondecreasing sequence in $\overline{B\left(x_{0}, r\right)}$ such that $x_{n} \rightarrow x \in \overline{B\left(x_{0}, r\right)}$ as $n \rightarrow+\infty$, then $x_{n} \preceq x$ for all $n \in \mathbb{N}$.

Then $S$ and $T$ have a common fixed point.

Corollary 3.9 Let $\left(X, d_{l}, \preceq\right)$ be a preordered complete dislocated metric space and let the pair $(S, T)$ of self-maps of $X$ be jointly nondecreasing and $x_{0} \in \overline{B\left(x_{0}, r\right)}$. Suppose that the following assertions hold:

(i) there exists $k \in[0,1)$ such that $d_{l}(S x, T y) \leq k d_{l}(x, y)$ for all $x, y \in \overline{B\left(x_{0}, r\right)}$ with $x \leq y$;

(ii) $x_{0} \preceq S x_{0}$ and $d_{l}\left(x_{0}, S x_{0}\right) \leq(1-k) r$;

(iii) if $\left\{x_{n}\right\}$ is a nondecreasing sequence in $\overline{B\left(x_{0}, r\right)}$ such that $x_{n} \rightarrow x \in \overline{B\left(x_{0}, r\right)}$ as $n \rightarrow+\infty$, then $x_{n} \preceq x$ for all $n \in \mathbb{N}$.

Then $S$ and $T$ have a common fixed point.

Corollary 3.10 Let $\left(X, d_{l}, \preceq\right)$ be a preordered complete dislocated metric space and let the pair $(S, T)$ of self-maps of $X$ be jointly nondecreasing. Suppose that the following assertions hold:

(i) there exists $k \in[0,1)$ such that $d_{l}(S x, T y) \leq k d_{l}(x, y)$ for all $x, y \in X$ with $x \preceq y$;

(ii) $x_{0} \preceq S x_{0}$;

(iii) if $\left\{x_{n}\right\}$ is a nondecreasing sequence in $X$ such that $x_{n} \rightarrow x \in X$ as $n \rightarrow+\infty$, then $x_{n} \preceq x$ for all $n \in \mathbb{N}$.

Then $S$ and $T$ have a common fixed point.

Corollary 3.11 Let $\left(X, d_{l}, \preceq\right)$ be a preordered complete dislocated metric space and $S: X \rightarrow$ $X$ be a nondecreasing map and $x_{0} \in \overline{B\left(x_{0}, r\right)}$. Suppose that the following assertions hold: 
(i) there exists $k \in[0,1)$ such that $d_{l}(S x, S y) \leq k d_{l}(x, y)$ for all $x, y \in \overline{B\left(x_{0}, r\right)}$ with $x \leq y$;

(ii) $x_{0} \preceq S x_{0}$ and $d_{l}\left(x_{0}, S x_{0}\right) \leq(1-k) r$;

(iii) if $\left\{x_{n}\right\}$ is a nondecreasing sequence in $\overline{B\left(x_{0}, r\right)}$ such that $x_{n} \rightarrow x \in \overline{B\left(x_{0}, r\right)}$ as $n \rightarrow+\infty$, then $x_{n} \preceq x$ for all $n \in \mathbb{N}$.

Then $S$ has a fixed point.

Corollary 3.12 Let $\left(X, d_{l}, \preceq\right)$ be a preordered complete dislocated metric space and $S$ : $X \rightarrow X$ be a nondecreasing map. Suppose that the following assertions hold:

(i) there exists $k \in[0,1)$ such that $d_{l}(S x, S y) \leq k d_{l}(x, y)$ for all $x, y \in X$ with $x \preceq y$;

(ii) there exists $x_{0} \in X$ such that $x_{0} \preceq S x_{0}$;

(iii) if $\left\{x_{n}\right\}$ is a nondecreasing sequence in $X$ such that $x_{n} \rightarrow x \in X$ as $n \rightarrow+\infty$, then $x_{n} \preceq x$ for all $n \in \mathbb{N}$.

Then $S$ has a fixed point.

Corollary 3.13 [27] Let $(X, d, \preceq)$ be a preordered complete metric space and $S: X \rightarrow X$ be a nondecreasing mapping such that

$$
d(S x, S y) \leq k d(x, y)
$$

for all $x, y \in X$ with $x \leq y$ where $0 \leq k<1$. Suppose that the following assertions hold:

(i) there exists $x_{0} \in X$ such that $x_{0} \preceq S x_{0}$;

(ii) if $\left\{x_{n}\right\}$ is a sequence in $X$ such that $x_{n} \preceq x_{n+1}$ for all $n \in \mathbb{N}$ and $x_{n} \rightarrow x$ as $n \rightarrow+\infty$, then $x_{n} \preceq x$ for all $n \in \mathbb{N}$.

Then $S$ has a fixed point.

Remark 3.14 We can similarly obtain partial metric and preordered partial metric versions of all results proved here which provide new results in the literature.

\section{Competing interests}

The authors declare that they have no competing interests.

Authors' contributions

All authors contributed equally and significantly in writing this article. All authors read and approved the final manuscript.

\section{Author details}

'Department of Mathematics, King Abdulaziz University, P.O. Box 80203, Jeddah, 21589, Saudi Arabia. ${ }^{2}$ Department of Mathematics, International Islamic University, H-10, Islamabad, 44000, Pakistan.

\section{Acknowledgements}

This article was funded by the Deanship of Scientific Research (DSR), King Abdulaziz University, Jeddah. Therefore, the first author acknowledges with thanks DSR, KAU for financial support.

Received: 26 December 2013 Accepted: 14 March 2014 Published: 31 Mar 2014

\section{References}

1. Abbas, $M$, Nazir, T: Common fixed point of a power graphic contraction pair in partial metric spaces endowed with a graph. Fixed Point Theory and Applications 2013, 20 (2013)

2. Abdeljawad, T: Meir-Keeler $\alpha$-contractive fixed and common fixed point theorems. Fixed Point Theory Appl. 2013, 19 (2013)

3. Agarwal, RP, El-Gebeily, MA, O’Regan, D: Generalized contractions in partially ordered metric spaces. Appl. Anal. 87, $109-116(2008)$

4. Agarwal, RP, Hussain, N, Taoudi, MA: Fixed point theorems in ordered Banach spaces and applications to nonlinear integral equations. Abstr. Appl. Anal. 2012, Article ID 245872 (2012)

5. Amini-Harandi, A: Metric-like spaces, partial metric spaces and fixed points. Fixed Point Theory Appl. 2012, 204 (2012) 
6. Amini-Harandi, A, Fakhar, M, Hajisharifi, HR, Hussain, N: Some new results on fixed and best proximity points in preordered metric spaces. Fixed Point Theory Appl. 2013, 263 (2013)

7. Arshad, M, Azam, A, Vetro, P: Some common fixed point results in cone metric spaces. Fixed Point Theory Appl. 2009, Article ID 493965 (2009)

8. Arshad, M, Shoaib, A, Beg, l: Fixed point of a pair of contractive dominated mappings on a closed ball in an ordered complete dislocated metric space. Fixed Point Theory Appl. 2013, 115 (2013)

9. Arshad, M, Shoaib, A, Vetro, P: Common fixed points of a pair of Hardy-Rogers type mappings on a closed ball in ordered dislocated metric spaces. J. Funct. Spaces Appl. 2013, Article ID 638181 (2013)

10. Bhaskar, TG, Lakshmikantham, V: Fixed point theorems in partially ordered metric spaces and applications. Nonlinear Anal. 65, 1379-1393 (2006)

11. Bojor, F: Fixed point theorems for Reich type contraction on metric spaces with a graph. Nonlinear Anal. 75 3895-3901 (2012)

12. Bojor, F: Fixed point of $\varphi$-contraction in metric spaces endowed with a graph. An. Univ. Craiova, Ser. Mat. Inform. 37(4), 85-92 (2010)

13. Ćirić, L, Abbas, M, Saadati, R, Hussain, N: Common fixed points of almost generalized contractive mappings in ordered metric spaces. Appl. Math. Comput. 217, 5784-5789 (2011)

14. Azam, A, Hussain, S, Arshad, M: Common fixed points of Chatterjea type fuzzy mappings on closed balls. Neural Comput. Appl. 21(Suppl 1), S313-S317 (2012)

15. Azam, A, Waseem, M, Rashid, M: Fixed point theorems for fuzzy contractive mappings in quasi-pseudo-metric spaces Fixed Point Theory Appl. 2013, 27 (2013)

16. Harjani, J, Sadarangani, K: Generalized contractions in partially ordered metric spaces and applications to ordinary differential equations. Nonlinear Anal. 72(3-4), 1188-1197 (2010)

17. Hitzler, P, Seda, AK: Dislocated topologies. J. Electr. Eng. 51(12/s), 3-7 (2000)

18. Hussain, N, Salimi, P, Latif, A: Fixed point results for single and set-valued $\alpha-\eta-\psi$-contractive mappings. Fixed Point Theory Appl. 2013, 212 (2013)

19. Hussain, N, Karapinar, E, Salimi, P, Vetro, P: Fixed point results for $G^{m}$-Meir-Keeler contractive and G- $(\alpha, \psi)$-Meir-Keeler contractive mappings. Fixed Point Theory Appl. 2013, 34 (2013)

20. Hussain, N, Karapinar, E, Salimi, P, Akbar, F: $\alpha$-admissible mappings and related fixed point theorems. J. Inequal. Appl. 2013, $114(2013)$

21. Hussain, N, Al-Mezel, S, Salimi, P: Fixed points for $\psi$-graphic contractions with application to integral equations. Abstr. Appl. Anal. 2013, Article ID 575869 (2013)

22. Hussain, N, Khan, AR, Agarwal, RP: Krasnosel'skii and Ky Fan type fixed point theorems in ordered Banach spaces. J. Nonlinear Convex Anal. 11(3), 475-489 (2010)

23. Hussain, N, Taoudi, MA: Krasnosel'skii-type fixed point theorems with applications to Volterra integral equations. Fixed Point Theory Appl. 2013, 196 (2013)

24. Jachymski, J: The contraction principle for mappings on a metric space with a graph. Proc. Am. Math. Soc. 1(136), 1359-1373 (2008)

25. Kutbi, MA, Ahmad, J, Hussain, N, Arshad, M: Common fixed point results for mappings with rational expressions. Abstr. Appl. Anal. 2013, Article ID 549518 (2013)

26. Matthews, SG: Partial metric topology. Proc. 8th Summer Conference on General Topology and Applications. Ann. N.Y. Acad. Sci. 728, 183-197 (1994)

27. Nieto, JJ, Rodríquez-López, R: Contractive mapping theorems in partially ordered sets and applications to ordinary differential equations. Order 22, 223-229 (2005)

28. Ran, ACM, Reurings, MCB: A fixed point theorem in partially ordered sets and some applications to matrix equations. Proc. Am. Math. Soc. 132, 1435-1443 (2003)

29. Ren, Y, Li, J, Yu, Y: Common fixed point theorems for nonlinear contractive mappings in dislocated metric spaces. Abstr. Appl. Anal. 2013, Article ID 483059 (2013)

30. Roldán, A, Karapınar, E: Some multidimensional fixed point theorems on partially preordered $G^{*}$-metric spaces under $(\psi, \varphi)$-contractivity conditions. Fixed Point Theory Appl. 2013, 158 (2013)

31. Salimi, P, Latif, A, Hussain, N: Modified $\alpha-\psi$-contractive mappings with applications. Fixed Point Theory Appl. 2013 $151(2013)$

32. Samet, B, Vetro, C, Vetro, P: Fixed point theorems for $\alpha$ - $\psi$-contractive type mappings. Nonlinear Anal. 75, 2154-2165 (2012)

33. Shoaib, A, Arshad, M, Ahmad, J: Fixed point results of locally cotractive mappings in ordered quasi-partial metric spaces. Sci. World J. 2013, Article ID 194897 (2013)

10.1186/1029-242X-2014-136

Cite this article as: Hussain et al.: Common fixed point results for $\alpha-\psi$-contractions on a metric space endowed with graph. Journal of Inequalities and Applications 2014, 2014:136 